\title{
Initial Experience of Coil Embolization Original for Unruptured Intracranial Aneurysm Combined with Neuroform Atlas and Undersized Flexible Coils
}

Koji Omoto, ${ }^{1}$ Katsutoshi Takayama, ${ }^{2}$ Ai Okamoto, ${ }^{1}$ Kaoru Myochin, ${ }^{3}$ Takeshi Wada, ${ }^{2}$ Ichiro Nakagawa, ${ }^{4}$ Shinichiro Kurokawa, ${ }^{1}$ Hiroyuki Nakase, ${ }^{4}$ and Kimihiko Kichikawa ${ }^{3}$

Objective: Intraprocedural rupture (IPR) is a rare complication that can occur during endovascular treatment (EVT) of unruptured intracranial aneurysms (UIAs). However, it leads to high morbidity and mortality rates. Others have showed that coil flexibility is a risk factor for IPR. Neuroform Atlas (NA) stents can be deployed with 0.0165 -inch microcatheters to enable stent assisted coiling (SAC) with a high likelihood. Undersized flexible coils can be inserted initially during SAC. This study aimed to determine whether SAC using NA and highly flexible coils for UIAs can be conducted without IPR.

Methods: We retrospectively analyzed nine consecutive patients (mean age, 73.2 years; female, $n=6$ ) who underwent SAC for UIAs combined with NA stents and undersized flexible coils between January 2017 and December 2019. Two aneurysms were located at the internal carotid artery (ICA), and one each was located at the ICA-posterior communicating, anterior communicating, middle cerebral, vertebral, vertebra-posterior inferior cerebral and basilar arteries. The mean size of the aneurysms was 4.6 (range, 3.1-8.6) $\mathrm{mm}$. SAC proceeded using the jailing technique. All coils were selected from among the most flexible coils available. We retrospectively assessed technical success rates, aneurysm occlusion at final digital subtraction angiography (DSA), volume embolization ratios (VERs), rates of IPR and symptomatic stroke within 30 days, angiographic findings of aneurysm occlusion at 3 months post-procedure and late adverse events (frequency of aneurysmal rupture, ipsilateral ischemic stroke, and retreated targeted aneurysms).

Results: The technical success rate was $100 \%$. Complete occlusion (CO) was immediate in $8(89 \%)$ patients and a neck remnant persisted in 1 (11\%). No IPR or symptomatic stroke developed within 30 days. During a mean follow-up period of 11.8 months, CO persisted in $8(89 \%)$ patients. No late adverse events occurred.

Conclusion: The early clinical and angiographic findings of SAC for UIAs combined with an NA stent and undersized flexible coils were favorable for this series.

Keywords $>$ unruptured intracranial aneurysm, Neuroform Atlas, coil embolization

${ }^{1}$ Departments of Neurosurgery, Ishinkai Yao General Hospital, Yao, Osaka, Japan

${ }^{2}$ Departments of Radiology and Interventional Radiology, Ishinkai Yao General Hospital, Yao, Osaka, Japan

${ }^{3}$ Department of Radiology, Nara Medical University, Kashihara, Nara, Japan

${ }^{4}$ Department of Neurosurgery, Nara Medical University, Kashihara, Nara, Japan

Received: March 26, 2020; Accepted: July 20, 2020

Corresponding author: Koji Omoto. Department of Neurosurgery, Ishinkai Yao General Hospital, 1-41, Numa, Yao, Osaka 581-0036, Japan

Email: koujiplain128@gmail.com

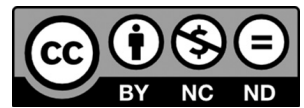

This work is licensed under a Creative Commons Attribution-NonCommercialNoDerivatives International License.

(C)2021 The Japanese Society for Neuroendovascular Therapy

\section{Introduction}

Intraprocedural rupture (IPR) is a rare complication that can occur during endovascular treatment (EVT) of unruptured intracranial aneurysms (UIAs). However, it leads to high morbidity and mortality rates. ${ }^{1}$ The rates of IPR for UIAs in the Japanese Registry of Neuroendovascular Therapy (JR-NET) and JR-NET2 during 2007 and 2010 using any adjunctive technique except a Flow Diverter, was $1.4 \%$ per aneurysm and procedure. ${ }^{2)}$ Lamano et al. ${ }^{3)}$ also reported that the force on the aneurysm wall associated with coil insertion is typically associated with coil flexibility. This could indicate that coil flexibility, the first in particular, is a risk factor for IPR. According to Murayama et al., ${ }^{4)}$ aneurysm perforation is more likely to be fatal when it occurs during the early 
Table 1 The characteristics of the patients and aneurysms

\begin{tabular}{|c|c|c|c|c|c|c|}
\hline & Age & Gender & $\begin{array}{l}\text { Aneurysm } \\
\text { size (mm) }\end{array}$ & $\begin{array}{c}\text { Neck } \\
\text { width }(\mathrm{mm})\end{array}$ & $\begin{array}{l}\text { Dome-to- } \\
\text { neck ratio }\end{array}$ & $\begin{array}{c}\text { Aneurysmal } \\
\text { location }\end{array}$ \\
\hline Case 1 & 83 & Male & 8.2 & 6.1 & 1.34 & MCA \\
\hline Case 2 & 77 & Female & 7.7 & 7.7 & 1.0 & ICA \\
\hline Case 3 & 81 & Female & 3.2 & 3.5 & 0.62 & A-com \\
\hline Case 4 & 79 & Female & 3.1 & 2.1 & 1.38 & VA-PICA \\
\hline Case 5 & 78 & Female & 4.6 & 3.3 & 0.85 & BA \\
\hline Case 6 & 39 & Female & 6.1 & 5.0 & 1.22 & ICA \\
\hline Case 7 & 76 & Female & 4 & 2 & 2.0 & VA-PICA \\
\hline Case 8 & 76 & Male & 4.9 & 4.1 & 1.2 & VA \\
\hline Case 9 & 76 & Male & 3.1 & 2.9 & 0.9 & ICA-Pcom \\
\hline
\end{tabular}

phase of EVT. Thus, IPR with the first coil must be avoided as it universally leads to life-threatening outcomes. ${ }^{5)}$

The Neuroform Atlas (NA) stent (Stryker, Kalamazoo, MI, USA) was approved in Japan during 2017. This is the first low-profile open-cell stent with a hybrid design. It has closed cells at the proximal ends to improve stability within the vessel and facilitate micro catheter re-crossing, and a central open-cell design to improve wall apposition and flexibility. Because the NA stent can be delivered through a 0.0165 -inch microcatheter and has good wall apposition and stability, complex UIAs with a parent vessel that is difficult to access due to tortuosity or a small diameter can be treated by stent-assisted coiling (SAC). Thus, a stiff framing coil is not necessarily required for SAC as the stent covers the aneurysmal neck to avoid coil protrusion into the parent artery. Highly flexible coils can be inserted during SAC as the first coil. The safety and outcomes of EVT of UIAs combined with NA and undersized flexible coils have yet to be assessed. The present study aimed to determine whether SAC using NA and undersized flexible coils to treat UIAs could avoid IPR and result in favorable occlusion outcomes.

\section{Materials and Methods}

\section{Patient population}

Among 34 patients with a total of 34 unruptured aneurysms that were treated by coil embolization at our hospital between January 2017 and December 2019, we retrospectively analyzed data from nine consecutive patients who had aneurysms (mean age, 73.2 years; range, 39-81; female, $\mathrm{n}=6$ ) that were embolized using NA stents and undersized flexible coils. All patients were assessed by diagnostic digital subtraction angiography (DSA) before the procedure to evaluate the morphovolumetric characteristics of the aneurysms. Treatment of UIAs in our center is performed after careful assessment of perceived risk factors for rupture. Neurosurgeons and neuro-radiologists discuss the decision of coiling or clipping. Inclusion criteria for SAC were as follows: (1) wide-necked saccular aneurysm, defined as an aneurysm with a neck dimension $\geq 4 \mathrm{~mm}$ or a dome-to-neck ratio $<2$; (2) parent vessel diameter $\geq 2 \mathrm{~mm}$; and (3) morphology or size of the aneurysm considered to indicate difficulty of treatment using balloon remodeling techniques. The mean aneurysmal size and dome-to-neck ratios calculated from DSA findings were $5.0 \pm 1.8$ (range, 3.1-8.2) $\mathrm{mm}$ and $1.19 \pm 0.40$ (range, $0.6-2.0$ ). Two aneurysms were located in the internal carotid artery (ICA) and one each was located in the ICA-posterior communicating, anterior communicating, middle cerebral, vertebral, vertebral-posterior inferior cerebral (VA-PICA) and basilar arteries. Table 1 shows the list for the baseline characteristics of the patients and aneurysms. Ethical approval was obtained from the institutional review board at our hospital (Approval No. 1003) and written informed consent was obtained from all patients before undergoing the procedures.

\section{Antiplatelet therapy}

All patients underwent dual-antiplatelet therapy (DAPT) for 14 days before the endovascular procedure with a loading dose of acetylsalicylic acid (ASA) $300 \mathrm{mg}$ and clopidogrel (CLP) $300 \mathrm{mg}$ on day 1 followed by ASA $100 \mathrm{mg}$ and CLP $75 \mathrm{mg}$ from day 2. We tested platelet function using the Verify Now assay system (Accumetrics, San Diego, CA, USA) to ensure a good response to ASA and CLP at 7 days before the procedure. A good response was defined as $\leq 550$ aspirin reaction units (ARU) and $\leq 200 \mathrm{P} 2$ Y 12 reaction units (PRU). Hypo-responders (PRU $\geq 200$ ) to CLP were switched 
from CLP to prasugrel $(20 \mathrm{mg})$ on day 1 and maintained on $3.75 \mathrm{mg} /$ day or cilostazol $200 \mathrm{mg} /$ day. The patients were continued on DAPT for 1 month post-procedure and single-antiplatelet therapy was continued for at least two months until follow-up assessment by DSA as described.6)

\section{Endovascular procedures}

All procedures were conducted under general anesthesia. The femoral artery was accessed through an 8Fr long sheath. Systemic heparinization (100 IU/kg) was initiated immediately after a femoral introducer sheath was inserted and activated clotting time (ACT) was maintained at 250 300 s. An $8 \mathrm{Fr}$ or a $6 \mathrm{Fr}$ guide catheter was placed proximally into the internal carotid or vertebral artery. A $6 \mathrm{~F}$ distal access catheter was navigated to the petrous or cavernous sinus portion of the ICA if anterior circulation was found. A microcatheter was placed into the aneurysmal sac, then a 0.014 neurovascular microguidewire was crossed over the aneurysmal neck using an Excelsior SL-10 microcatheter (Stryker). An NA stent was deployed across the aneurysmal neck with a jailed intra-aneurysm microcatheter. The stent size was selected based on the size of the parent artery determined by diagnostic DSA. The aneurysm was coiled with a flexible coil via the jailed microcatheter in the same series. All coils were selected from one of the most flexible coil series (HyperSoft) produced by one manufacturer (MicroVention, Aliso Viejo, CA, USA) and Axium Prime Extra Soft (Medtronic, Dublin, Ireland). How to choose the size and length of coils was as follows: when the maximum diameter of the aneurysm was larger than $5 \mathrm{~mm}$, we chose as the first coil at least $2 \mathrm{~mm}$ smaller coil diameter than the maximum diameter and the longest coil among the same diameter coils we had chosen. When the maximum diameter of aneurysm was smaller than $4 \mathrm{~mm}$, basically we chose $2 \mathrm{~mm} \times 4 \mathrm{~cm}$ or $3 \mathrm{~cm}$ coil. Then, the aneurysm was coiled with $2 \mathrm{~mm} \times 2 \mathrm{~cm}$ as much as possible until the jailed microcatheter dropped out of the aneurysmal neck or just before coils protruded into the parent artery. When it was difficult to be coiled with $2 \mathrm{~mm} \times$ $2 \mathrm{~cm}$, we chose $1.5 \mathrm{~mm} \times 2 \mathrm{~cm}$ or $1 \mathrm{~mm} \times 2 \mathrm{~cm}$. Our strategy of coil selection was similar to the so-called piecemeal technique. ${ }^{7)}$

\section{Endpoints and definitions}

The primary endpoints were periprocedural outcomes within 30 days. We also assessed technical success rates, aneurysm occlusion using the Raymond-Roy Occlusion Classification (RROC) at final DSA, volume embolization ratios (VERs) and complication rates (frequency of IPR and ipsilateral symptomatic stroke). Technical success was defined as successful placement of NA for targeted aneurysmal neck and placement of coils into the aneurysmal sac. Aneurysm occlusion was classified according to RROC as complete occlusion (CO), residual neck (NR), and residual aneurysm (RA). An expert neuroradiologist who was not involved in the procedures retrospectively assessed the RROC data. The VER was calculated using: $\mathrm{V}_{\text {coil }} / \mathrm{V}_{\text {aneurysm }} \times 100(\%)\left[\mathrm{V}_{\text {coil }}=\pi \times(\mathrm{p} / 2)^{2} \times \mathrm{L}\right.$ and $\mathrm{V}_{\text {aneurysm }}=$ $4 / 3 \pi \times$ length $/ 2 \times$ width $/ 2 \times$ height $/ 2$, where $p$ is the primary diameter of the coil and $\mathrm{L}$ is the coil length as described $\left.^{8}\right]$ ]. Symptomatic stroke was defined as a neurological deficit that persisted for $>24$ hours. Secondary endpoints were late outcomes from 30 days after the procedure (follow-up). We assessed aneurysm occlusion classified by the RROC using DSA 3 months after the procedure and the rate of adverse events (frequency of aneurysmal rupture, ipsilateral stroke, and re-treated targeted aneurysms).

\section{Results}

Table 2 shows the list of the procedure characteristics and periprocedural outcome. The technical success rate was 100\% (Fig. 1C-1F). The NA stents were appropriately deployed except one patient whose aneurysm was located at VA-PICA. In this case, the first stent was migrated to proximal neck of the aneurysm, then additional stent was placed appropriately. Coils were placed in all aneurysms. Axium Prime Extra Soft and HyperSoft coils were placed in two and seven aneurysms, respectively. The jailing technique was applied during all procedures. The sizes of the deployed NA stents were $3.0 \times 21(\mathrm{n}=2), 4.0 \times 21(\mathrm{n}=6)$, and $4.5 \times 21(\mathrm{n}=1) \mathrm{mm}$, respectively.

CO was immediate in eight (89\%) patients (Fig. 1G), and an NR remained in one (11\%). No RA, IPR or symptomatic stroke occurred and the VER was $55.8 \% \pm$ $13.9 \%$.

During a mean follow-up period of 11.8 (range, 4-30) months, $\mathrm{CO}$ was achieved in eight patients (Fig. $\mathbf{1 H})$, with NR remaining in a patient at the time of follow-up DSA to assess aneurysm occlusion. No major adverse events developed.

\section{Discussion}

Outcome and follow-up data for treatment of intracranial aneurysms using NA stents in the present and several other 
Table 2 The characteristics of the procedure and periprocedural outcome

\begin{tabular}{lcccccc} 
& $\begin{array}{c}\text { Coil } \\
\text { series }\end{array}$ & $\begin{array}{c}\text { NA stent } \\
\text { dimensions }(\mathrm{mm})\end{array}$ & $\begin{array}{c}\text { Technical } \\
\text { success }\end{array}$ & RROC & IPR & VER (\%) \\
Case 1 & AP & $3.0 \times 21$ & Yes & NR & No & 32 \\
Case 2 & AP & $4.0 \times 21$ & Yes & CO & No & 57.6 \\
Case 3 & HS & $3.0 \times 21$ & Yes & CO & No & 40.7 \\
Case 4 & HS & $4.0 \times 21$ & Yes & CO & No & 49.7 \\
Case 5 & HS & $4.0 \times 21$ & Yes & CO & No & 77 \\
Case 6 & HS & $4.0 \times 21$ & Yes & CO & No & 54 \\
Case 7 & HS & $4.0 \times 21$ & Yes & CO & No & 57.6 \\
Case 8 & HS & $4.0 \times 21$ & Yes & CO & No & 56.1 \\
Case 9 & HS & $4.5 \times 21$ & Yes & CO & No & 76.7 \\
\hline
\end{tabular}

Coil series: AP: Axium Prime Extra Soft (Medtornic); HS: Hyper Soft (Termo); NA: Neuroform Atlas; RROC: Raymond--Roy Occlusion Classification; CO: complete occlusion; NR: residual neck; RA: residual aneurysm; IPR: intraprocedural rupture; VER: volume embolization ratio
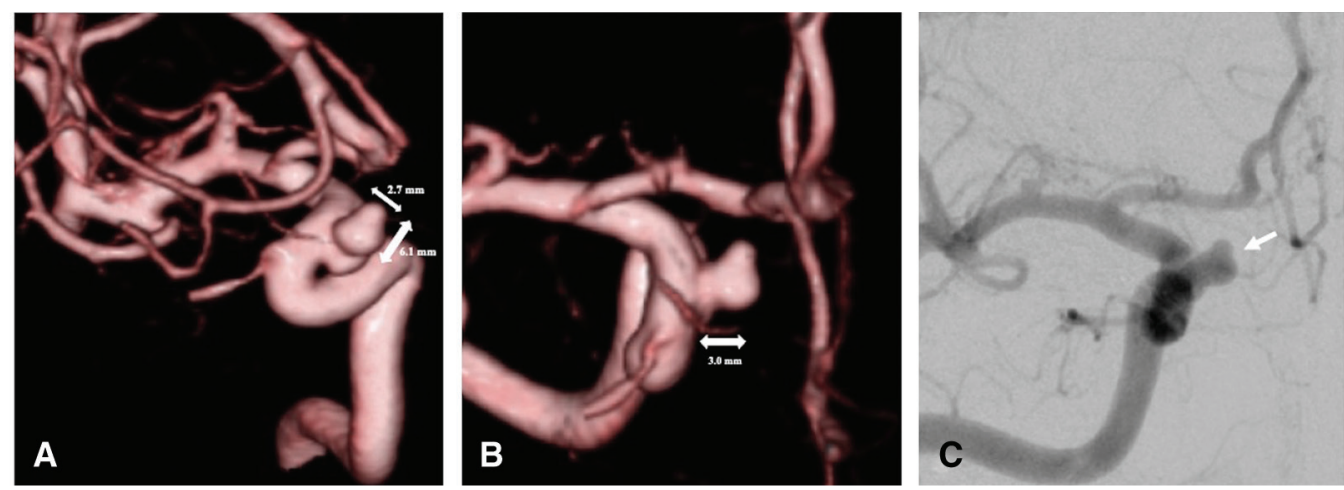
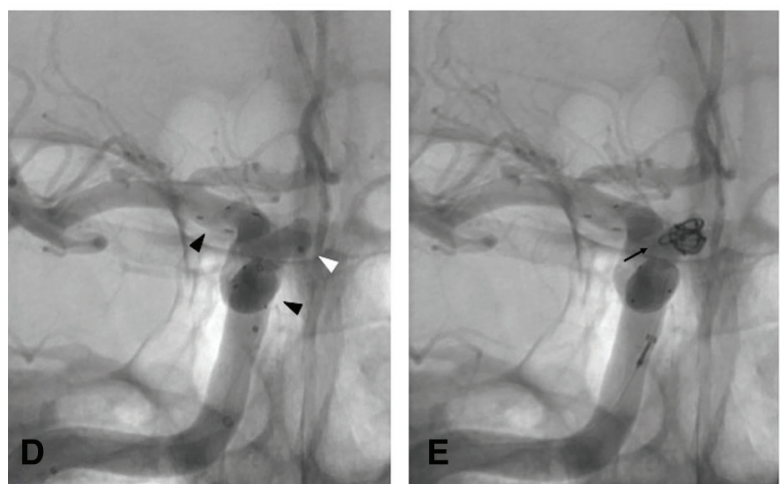

Fig. 1 Representative coil embolization with jailing technique combined with NA stent and flexible coil. The patient was 39-year-old female and was incidentally pointed out right ICA aneurysm with magnetic resonance imaging. (A) Diagnostic three-dimensional DSA shows the length of the aneurysm was $6.1 \mathrm{~mm}$ (large white double-headed arrow), that was the maximum diameter. The width of the aneurysm is $2.7 \mathrm{~mm}$ (small white double-headed arrow). (B) Diagnostic three-dimensional DSA shows the height of the aneurysm was $3.0 \mathrm{~mm}$ (white double-headed arrow). (C) Pretreatment right carotid angiography for unruptured right ICA aneurysm. (D) Unsubtracted image. Right carotid angiography shows neuroform atlas stent (black arrowhead) covering aneurysm neck appropriately jailed with microcatheter (white arrow head) for emboliza-

studies are summarized in Table 3. Although long-term occlusion and a need for retreatment are important outcomes of EVT for UIAs, avoiding IPR that leads to life-threatening
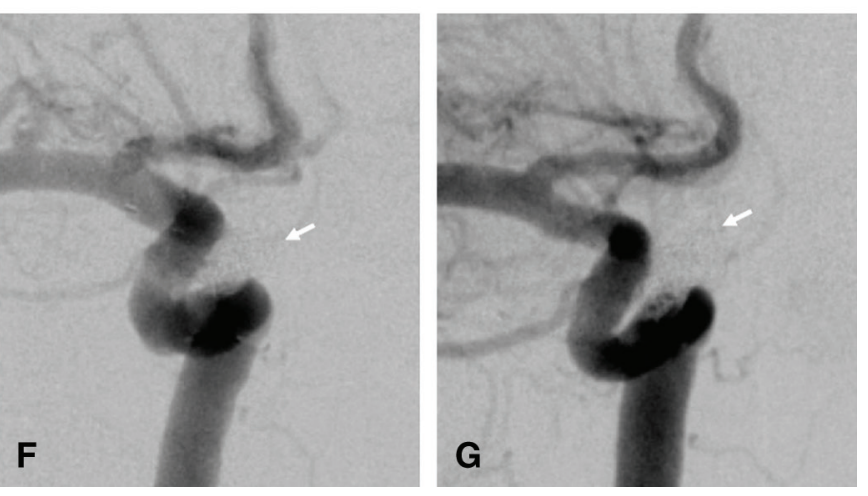

tion. (E) Unsubtracted image. Right carotid angiography shows that first HyperSoft ${ }^{\circledR}$ coil $(4.0 \mathrm{~mm} \times 8 \mathrm{~cm})$ is selected. First coil is placed without coil protrusion into parent artery (black arrow). (F) Right carotid angiogram demonstrates $\mathrm{CO}$ with tight packing (white arrow) using 9 coils (4.0

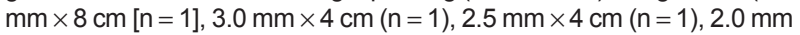
$\times 4 \mathrm{~cm}(\mathrm{n}=4), 1.5 \mathrm{~mm} \times 2 \mathrm{~cm}(\mathrm{n}=1)$, and $1.0 \times 2 \mathrm{~cm}(\mathrm{n}=1) \mathrm{mm}$, respectively). The measurement of volume embolization ratios is $54 \%$. The postoperative course was uneventful. The patient discharged a week after treatment without any complication. (G) Follow-up DSA at 3 months post-procedure demonstrates that occlusion remains complete without any symptoms (white arrow). CO: complete occlusion; DSA: digital subtraction angiography; ICA: internal carotid artery; NA: Neuroform Atlas

outcomes is most important.5) Focusing on IPR, A wide spectrum of IPR rates have been reported, and it is currently estimated to be between $1 \%$ and $5 \%{ }^{9,10)}$ Pierot et al. $\left.{ }^{9}\right)$ 


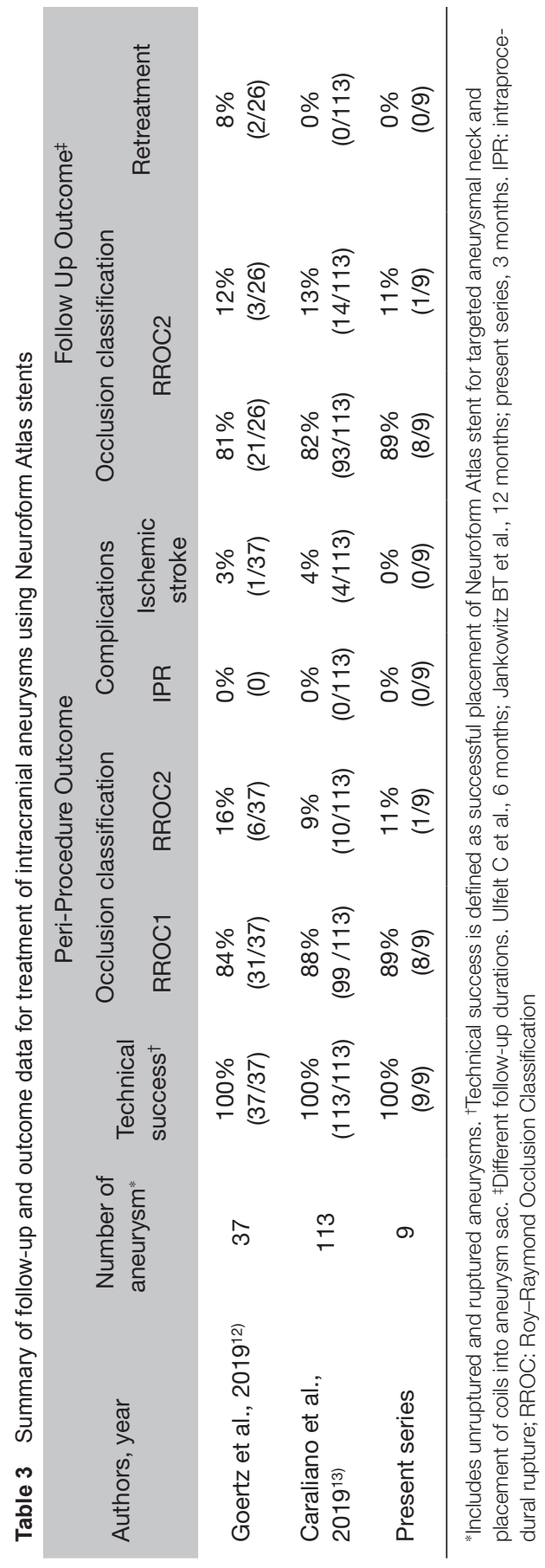

reported in the prospective multicenter study the rate of IPR at SAC was $1.7 \%(1 / 57)$. In addition, a retrospective analysis of 1312 patients with an UIA by Li et al. found a lower risk of IPR with, than without stent deployment, ${ }^{11)}$ indicating that $\mathrm{SAC}$ would be as a regarding adjunctive technique from the perspective view of preventing IPR.

In in vitro experiments, Lamano et al. ${ }^{3)}$ showed that framing coil deployment could potentially increase the force acting on the aneurysmal wall. This indicated that IPR is associated with coil flexibility, particularly with that of framing coils that tend to be stiff. Firm framing coils are not necessarily required under a neck bridge stent. Although flexible first coils can be used to avoid IPR, the outcomes of SAC with flexible coils have not been assessed as far as we can ascertain. Therefore, this present study was conducted and showed no IPR occurred in all cases.

The present and several other studies have found that NA stents were correctly deployed with $100 \%$ technical success. ${ }^{12,13)}$ Aydin et al. ${ }^{14)}$ reported a 97.5\% technical success rate of using Leo Baby stents for SAC, Poncyljusz et al. ${ }^{15)}$ had a $91 \%$ technical success rate with LVIS/LVIS Jr. stents, and Mocco et al. ${ }^{16)}$ described correct deployment of an Enterprize stent in $95 \%$ of patients. The rates of technical success for other low-profile or microstent series were similar, but not $100 \%$. SAC with flexible coils is inevitable for stent placement. NA was suitable stent for SAC with flexible coils. Then, stent design might be one cause of these differences. The NA stent has an open- and closed-cell design, which is supposed to offer the advantages of both cell types, unlike previous stents. Cay et al. ${ }^{17)}$ reported that open-cell stents should be characterized by relatively low radial force, good wall coverage, and low bending stiffness. A cell stent with a closed design provides stable adherence to the vessel wall. Furthermore, foreshortening is negligible for laser-cut stents, which allows for accurate placement. On the other hand, braided stents have the disadvantages of foreshortening and the possibility of an hourglass configuration during deployment, which results in incomplete neck bridging. However, stent migration occurred in one case of right VA-PICA aneurysm which stent placement was attempted from the right PICA to the right VA to spare PICA. In this case, the angle between the right PICA and the right VA was $\angle 80^{\circ}$, and the difference of diameter between right PICA and the right VA was about five times. The cause of stent migration may be associated with these factors. Furthermore, the NA is compatible with good accessibility, allowing deployment with conventional coiling with 0.0165-inch microcatheters. These advantages might result 
in excellent outcomes of even higher technical success rates. Although none of the coils unraveled in our patients, coils can unravel during stent-assisted procedures. ${ }^{18)}$ Because coils can become trapped in intracranial-stent stent struts regardless of stent design, physicians should consider the possibility of coil unraveling during embolization.

Goertz et al. ${ }^{12)}$ and Caragliano et al. ${ }^{13)}$ achieved immediate $\mathrm{CO}$ in $84 \%$ and $88 \%$ of aneurysmal occlusions, respectively, using NA, and $81 \%$ and $82 \%$ occlusion rates at 6 and 12 months of follow-up, respectively. We achieved immediate CO in $89 \%$ of aneurysmal occlusions (Table 3 ). These results were favorable compared with those for braided stents. A recent study achieved immediate $\mathrm{CO}$ in $85 \%$ of 78 patients treated with LVIS stents and $82 \% \mathrm{CO}$ at 6 months of angiographic follow-up. Our $\mathrm{CO}$ at 3 months of angiographic follow-up was $89 \%$. Retreatment rates in the two series of patients who were treated with $\mathrm{NA}^{12,13)}$ were 2 $(7.7 \%)$ of 26 and $0(0 \%)$ of 112 , respectively. The RROC status was intraoperative aneurysm remnant in two patients, and secondary SAC achieved CO. None of the patients treated with NA in the one series ${ }^{12)}$ who had a neck remnant required retreatment. None of our patients had an aneurysm remnant, which might be associated with the $0 \%$ retreatment rate. We assume that the long-term outcomes for our patients would be favorable compared with previous findings.

The rates of ischemic stroke complications were $2.7 \%-$ $6.2 \%$ among the series of patients treated with an NA. ${ }^{12,13,19)}$ None of our patients were complicated with ischemic stroke, perhaps because of how we managed antiplatelet therapy. The rates of morbidity and mortality including subarachnoid hemorrhage $(\mathrm{SAH})$ in other NA series were $0 \%-3.3 \%$ and $0 \%-2.65 \%,{ }^{12,13,19,20)}$ and were both $0 \%$ in one NA series with UIA. ${ }^{19)}$ Morbidity was associated with peri-interventional thromboembolic events, in which in-stent occlusion did not occur due to rupture. Mortality was mainly associated with the severity of SAH. No intraprocedural hemorrhage was reported. Among patients with UIA, permanent neurological deficits did not arise due to the interventions, and neurological death did not occur. The morbidity and mortality rates were $3.0 \%$ and $1.0 \%$ for the LVIS Jr stents, respectively, ${ }^{21)}$ and $1.4 \%$ and $0 \%$ for LVIS stents, respectively. ${ }^{22)}$ The SAC using NA and the most flexible coils seems less complicated as morbidity and mortality did not arise in the present study.

Yagi et al. ${ }^{23)}$ significantly associated a high VER with low recurrence of coil embolization. This study found that the recanalization rate of 57 aneurysms at 6 months after coil embolization was significantly lower in a group with a high $(\geq 25 \%)$ than a low $(<25 \%)$ VER (2 [8\%] of 25 vs. $21[66 \%]$ of 32). The VER in the present study was $55.8 \%$, which was twice as high as the reported optimal VER cutoff to reduce recanalization of $20 \%-25 \% .^{24,25)}$ None of our patients required recanalization, but they were assessed by DSA at only 3 months after the procedure. The higher VER in the present study might be associated with long-term stability.

In conclusion, the initial experience of SAC combined with an NA stent and undersized flexible coils to treat UIA resulted in complete technical success, no complications, high rates of complete periprocedural occlusion, and followup compliance. However, larger samples and longer-term follow-up should be needed to determine durability and the need for retreatment.

\section{Limitations of the Study}

This retrospective study included a small patient cohort and a short follow-up period. Late rebleeding and repeated treatment should be assessed during a longer follow-up period.

\section{Conclusion}

SAC for UIA using an NA stent and undersized flexible coils was associated with favorable early clinical outcomes and angiographic results.

\section{Ethical Approval}

All study patients signed a written authorization allowing access to their medical records for research purposes, and our institutional review board approved the research protocol (No. 1003).

\section{Informed Consent}

Written informed consent was obtained from all patients who participated in the study.

\section{Disclosure Statement}

The authors have no conflicts of interest to declare.

\section{References}

1) Cloft HJ, Kallmes DF: Cerebral aneurysm perforations complicating therapy with Guglielmi detachable coils: a meta-analysis. AJNR Am JNeuroradiol 2002; 23: 1706-1709.

2) Shigematsu T, Fujinaka T, Yoshimine T, et al: Endovascular therapy for asymptomatic unruptured intracranial 
aneurysms: JR-NET and JR-NET2 findings. Stroke 2013; 44: 2735-2742.

3) Lamano JB, Bushnell GG, Chen H, et al: Force characterization of intracranial endovascular embolization: coil type, microcatheter placement, and insertion rate. Neurosurgery 2014; 75: 707-715; discussion 715-706.

4) Murayama Y, Viñuela F, Duckwiler GR, et al: Embolization of incidental cerebral aneurysms by using the Guglielmi detachable coil system. J Neurosurg 1999; 90: 207-214.

5) Santillan A, Gobin YP, Greenberg ED, et al: Intraprocedural aneurysmal rupture during coil embolization of brain aneurysms: role of balloon-assisted coiling. AJNR Am J Neuroradiol 2012; 33: 2017-2021.

6) Wada T, Takayama K, Myouchin K, et al: Investigation of safe termination of antiplatelet therapy after LVIS stentassisted cerebral aneurysm coiling. JNET J Neuroendovasc Ther 2019; 13: 199-205.

7) Tsumoto $T$, Terada T, Yamaga H, et al: Endovascular coil embolization for cerebral aneurysms solely using ultrasoft coils. Interv Neuroradiol 2006; 12: 101-104.

8) Imai $\mathrm{T}$, Ohshima $\mathrm{T}$, Goto $\mathrm{S}$, et al: Comparison of clinical outcomes using 10 coil versus 14 coil in endovascular coil embolization for small and medium-sized intracranial aneurysms: efficacy of 14 coil. JNET J Neuroendovasc Ther 2016; 10: 196-200.

9) Pierot L, Spelle L, Vitry F, et al: Immediate clinical outcome of patients harboring unruptured intracranial aneurysms treated by endovascular approach: results of the ATENA study. Stroke 2008; 39: 2497-2504.

10) Brisman JL, Niimi Y, Song JK, et al: Aneurysmal rupture during coiling: low incidence and good outcomes at a single large volume center. Neurosurgery 2005; 57: 1103-1109; discussion 1103-1109.

11) Li L, Zhang X, Feng Z, et al: Risk factors for intraprocedural rupture in the endovascular treatment of unruptured intracranial aneurysms: a single-center experience with 1232 procedures. World Neurosurg 2019; 123: e9-e14.

12) Goertz L, Dorn F, Siebert E, et al: Safety and efficacy of the Neuroform Atlas for stent-assisted coiling of intracranial aneurysms: a multicenter experience. J Clin Neurosci 2019; 68: 86-91.

13) Caragliano AA, Papa R, Pitrone A, et al: The low-profile Neuroform Atlas stent in the treatment of wide-necked intracranial aneurysms - immediate and midterm results: an Italian multicenter registry. $J$ Neuroradiol 2020; 47: 421-427.

14) Aydin K, Arat A, Sencer S, et al: Stent-assisted coiling of wide-neck intracranial aneurysms using low-profile LEO baby stents: initial and midterm results. AJNR Am J Neuroradiol 2015; 36: 1934-1941.

15) Poncyljusz W, Biliński P, Safranow K, et al: The LVIS/LVIS Jr. stents in the treatment of wide-neck intracranial aneurysms: multicentre registry. J Neurointerv Surg 2015; 7: 524-529.

16) Mocco J, Snyder KV, Albuquerque FC, et al: Treatment of intracranial aneurysms with the Enterprise stent: a multicenter registry. J Neurosurg 2009; 110: 35-39.

17) Cay F, Peker A, Arat A: Stent-assisted coiling of cerebral aneurysms with the Neuroform Atlas stent. Interv Neuroradiol 2018; 24: 263-269.

18) Schütz A, Solymosi L, Vince GH, et al: Proximal stent fixation of fractured coils: technical note. Neuroradiology 2005; 47: 874-878.

19) Jankowitz BT, Hanel R, Jadhav AP, et al: Neuroform Atlas stent system for the treatment of intracranial aneurysm: primary results of the Atlas Humanitarian Device Exemption cohort. J Neurointerv Surg 2019; 11: 801-806.

20) Ulfert $C$, Pham M, Sonnberger $M$, et al: The Neuroform Atlas stent to assist coil embolization of intracranial aneurysms: a multicentre experience. J Neurointerv Surg 2018; 10: 1192-1196.

21) Shankar JJS, Quateen A, Weill A, et al: Canadian registry of LVIS Jr for treatment of intracranial aneurysms (CaRLA). J Neurointerv Surg 2017; 9: 849-853.

22) Zhang $X$, Zhong J, Gao H, et al: Endovascular treatment of intracranial aneurysms with the LVIS device: a systematic review. J Neurointerv Surg 2017; 9: 553-557.

23) Yagi K, Satoh K, Satomi J, et al: Evaluation of aneurysm stability after endovascular embolization with Guglielmi detachable coils: correlation between long-term stability and volume embolization ratio. Neurol Med Chir (Tokyo) 2005; 45: 561-565; discussion 565-566.

24) Ikeda G, Sonobe M, Kato N, et al: Correlation between rupture or retreatment and the volume embolization ratio after coil embolization. Surg Cerebr Stroke 2013; 41: 440-446. (in Japanese)

25) Uchiyama N, Kida S, Nomura M, et al: Significance of volume embolization ratio as a predictor of recanalization on endovascular treatment of cerebral aneurysms with guglielmi detachable coils. Interv Neuroradiol 2000; 6: 59-63. 